\title{
The occurrence of Ammothella longioculata (Faraggiana, 1940) (Arthropoda, Pycnogonida) in İzmir Bay (Aegean Sea, Turkey) and reported species from the bay
}

\section{Ammothella longioculata (Faraggiana, 1940) (Arthropoda, Pycnogonida)'nın İzmir Körfezi'nde (Ege Denizi, Türkiye) bulunuşu ve körfezden rapor edilmiş türler}

\section{Cengiz Koçak}

Koçak, C. (2020). The occurrence of Ammothella longioculata (Faraggiana, 1940) (Arthropoda, Pycnogonida) in İzmir Bay (Aegean Sea, Turkey) and reported species from the bay. Ege Journal of Fisheries and Aquatic Sciences, 37(4), 373-378. DOl:10.12714/egejfas.37.4.08

Abstract: Sampling studies in Izmir Bay revealed the occurrence of a pycnogonid species, Ammothella longioculata (Faraggiana, 1940). A. longioculata is reported only one time from the Turkish waters, up to now. The presence of this rare species is reported herein for the first time from the izmir Bay, and also second time from the Turkish waters. The distribution map of the species in the Mediterranean Sea is provided, together with photographs and line drawing of the species. Moreover, all of the early studies were reviewed on the pycnogonid fauna of Izmir Bay, distribution of each species, depth range, and type of substrate are given.

Keywords: Ammothella longioculata, Pycnogonida, İzmir Bay, Turkey, Mediterranean Sea

Öz: İzmir Körfezi'nde yapılan örnekleme çalışmaları, bir piknogonid tür olan Ammothella longioculata (Faraggiana, 1940)'nın varlı̆ı̆ıı ortaya koymuştur. A. longioculata bugüne kadar Türkiye sularından yalnızca bir kez rapor edilmiștir. Bu nadir türün varlığı İzmir Körfezi'nden ilk kez, Türkiye sularından ise ikinci kez bu çalışmada rapor edilmektedir. Türün Akdeniz'deki dağıımı, fotoğraf ve çizimi çalışmada sunulmuştur. Ayrıca, İzmir Körfezi'nin piknogonid faunası üzerine daha önce yapılmış çalışmaların tümü gözden geçirilmiş olup, her bir türün dağıımı, derinlik aralığı ve subsratum tipi verilmiştir.

Anahtar kelimeler: Ammothella longioculata, Pycnogonida, İzmir Körfezi, Türkiye, Akdeniz

\section{INTRODUCTION}

Ammothella longioculata (Faraggiana, 1940) is endemic to the Mediterranean Sea. This species had been reported eleven times before from the region (Faraggiana, 1940; Stock, 1958; Krapp, 1973; Arnaud, 1987; Schüller, 1989; Chimenz et al., 1993; Munilla and Nieto, 1999; Vignoli et al., 2006; Kocak and Katagan, 2007; Krapp et al., 2008, who reported for the first time this species from the Turkish waters). The present study provides a new locality for $A$. longioculata in the Aegean Sea, information about its distribution in the Mediterranean Sea, and reported pycnogoid species from the İzmir Bay.

\section{MATERIALS AND METHODS}

One ovigerous male specimen of the species was collected by snorkeling from Cystoseira mediterranea facies at one station in the upper infralittoral zone (1m depth) of Urla, İmir Bay (Figure 1). The sample was fixed in 5\% formaldehyde and later rinsed with fresh water and then preserved in $70 \%$ ethanol. The specimen sampled was examined under a stereomicroscope. The drawing was made with the aid of a drawing tube mounted on an Olympus CX31 compound microscope. The following papers were used for species identification: Faraggiana (1940), Krapp (1973), and Kocak and Katagan (2007). The specimen was stored in the ESFM (Museum of the Faculty of Fisheries, Ege University, İzmir).

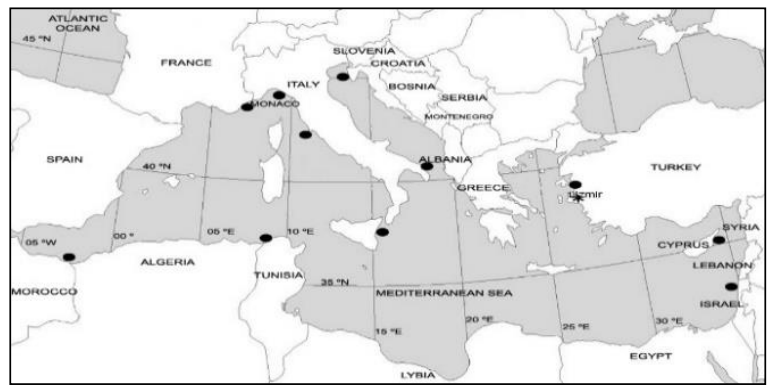

Figure 1. Distribution of Ammothella longioculata (Faraggiana, 1940) in the Mediterranean Sea $(\mathbf{O})$, including the sampling area $(*)$ 


\section{RESULTS}

\section{Systematics}

Class PYCNOGONIDA Latreille, 1810

Order PANTOPODA Gerstaecker, 1863

Family AMMOTHEIDAE Dohrn,1881

Genus Ammothella Verrill, 1900

Ammothella longioculata (Faraggiana, 1940) (Figures 2-3)

Material examined: 1 ovigerous $\hat{\partial}$, (EFSM-PYC/2007-1), Urla (İzmir Bay, Aegean Sea), 38 22'27"N, 26 47'13"E, Cystoseira mediterranea Sauvageau, 1912, 1 m, 04 July 2007.
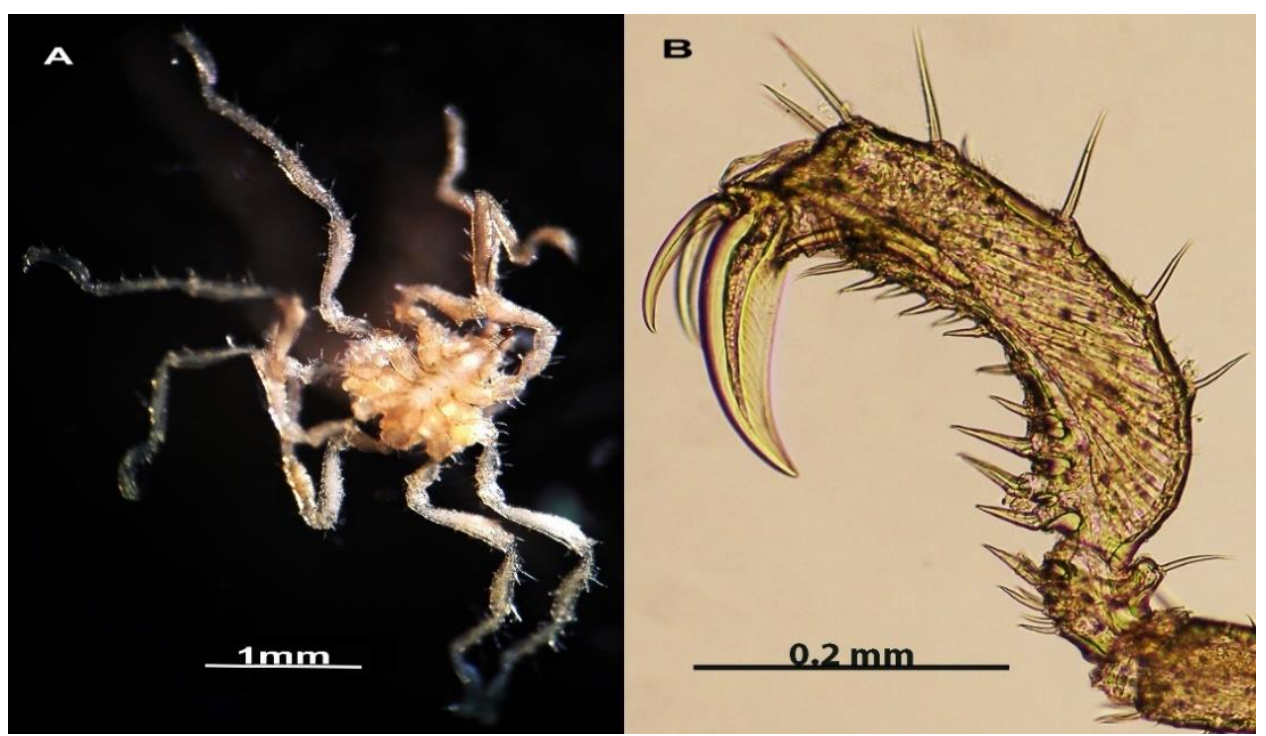

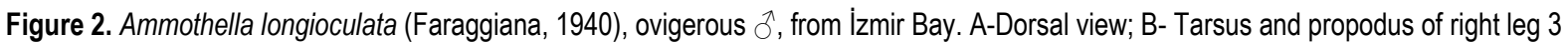

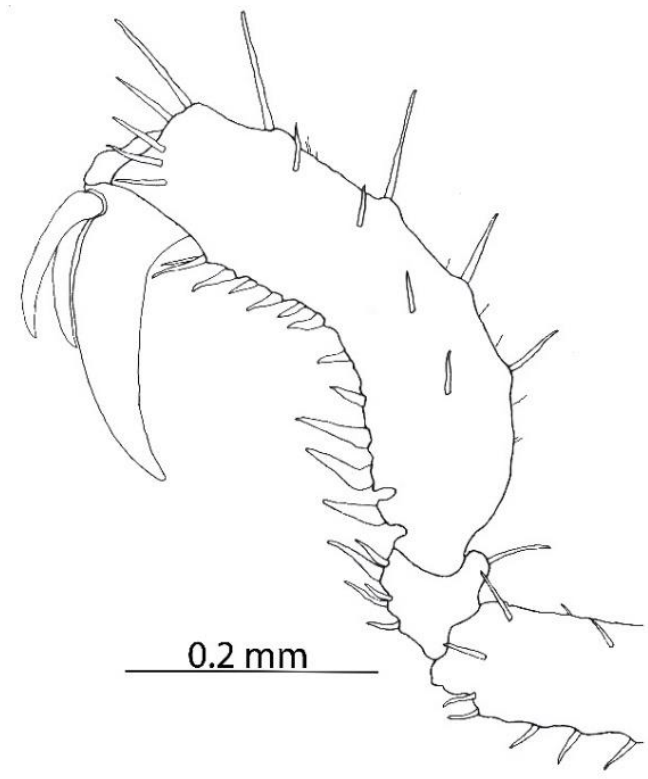

Figure 3. Ammothella longioculata (Faraggiana, 1940), ovigerous $\widehat{\jmath}$, from İzmir Bay. Line drawing of tarsus and propodus of right leg 3 
As a result of studies carried out to review the Pycnogonid fauna of the Izmir Bay, indicating the presence of 18 species inhabiting the Bay, belonging to 6 families and 9 genera:

SUPERFAMILY: ASCORHYNCHOIDEA POCOCK, 1904

FAMILY: AMMOTHEIDAE DOHRN, 1881

GENUS: AMMOTHELLA VERRILL, 1900

\section{A. appendiculata (Dohrn, 1881)}

Synonyms: Ammothea appendiculata Dohrn, 1881; Ammothea rugulosa Verrill, 1900

This species was reported by Çinar et al. (2008) from Alsancak Harbour, Pasaport Harbour, and İnciraltı.

Depth range: $0.2 \mathrm{~m}$.

Substrate: Mytilus galloprovincialis Lamarck, 1819.

Worldwide Distribution: Cosmopolitan (SolerMembrives and Munilla, 2015).

\section{A. longipes (Hodge, 1864)}

Synonyms: Achelia longipes (Hodge, 1864); Ammothea longipes Hodge, 1864; Ammothea magnirostris Dohrn, 1881; Ammothella magnirostris (Dohrn, 1881); Achelia hispida King, 1974

The species was reported by Arnaud (1976) from Kalabak, Mordoğan, and Urla.

Depth range: $0.5-1.5 \mathrm{~m}$.

Substrate: Cystoseira crinita Duby, 1830; Ellisolandia elongata (J. Ellis \& Solander) K.R. Hind \& G.W. Saunders, 2013 (as Corallina mediterranea J.E. Areschoug, 1852).

Worldwide Distribution: Atlantic-Mediterranean (SolerMembrives and Munilla, 2015).

\section{A. uniunguiculata (Dohrn, 1881)}

Synonyms: Ammothea uni-unguiculata Dohrn, 1881

This species was reported by Arnaud (1976) from Kalabak, Foça and Narlıdere, and Krapp et al. (2008) from Foça.

Depth range: $0.5-1.5 \mathrm{~m}$.

Substrate: C. crinata; Padina sp.; Ulva lactuca Linnaeus, 1753; Halopteris scoparia f. hiemalis J.Agardh.

Worldwide Distribution: Endemic (Mediterranean) (Soler-Membrives and Munilla, 2015).

GENUS: ACHELIA HODGE, 1864

\section{A. echinata Hodge, 1864}

Synonyms: Ammothea brevipes Hodge, 1864; Ammothea echinata (Hodge, 1864); Achelia fibulifera (Dohrn, 1881)
This species was reported by Arnaud (1976) from Kalabak (as Achelia cf. echinata).

Depth range: $0.5 \mathrm{~m}$.

Substrate: E. elongata (as C. mediterranea).

Worldwide Distribution: Cosmopolitan (SolerMembrives and Munilla, 2015).

\section{A. langi (Dohrn, 1881)}

Synonyms: Ammothea langi Dohrn, 1881

The species was reported by Krapp et al. (2008) from Foça.

Depth range: $0.5 \mathrm{~m}$.

Substrate: C. crinita.

Worldwide Distribution: Atlantic-Mediterranean (SolerMembrives and Munilla, 2015).

GENUS: TANYSTYLUM MIERS, 1879

T. conirostre (Dohrn, 1881)

Synonyms: Clotenia conirostris Dohrn, 1881

The species was reported by Arnaud (1976) from Kalabak, Narlıdere and Foça, Krapp et al. (2008) from Foça, and Çinar et al. (2008) Alsancak Harbour, Pasaport Harbour and İnciraltı.

Depth range: $0.2-1.5 \mathrm{~m}$.

Substrate: C. crinata; U. lactuca; E. elongata (as C. mediterranea); M. galloprovincialis.

Worldwide Distribution: Atlantic-Mediterranean (SolerMembrives and Munilla, 2015).

\section{T. orbiculare Wilson, 1878}

Synonyms: Clotenia orbiculare (Wilson, 1878)

This species was recorded by Arnaud (1976) from Kalabak, and Krapp et al. (2008) from Foça.

Depth range: $0.5-1 \mathrm{~m}$.

Substrate: E. elongata (as C. mediterranea); C. crinita; H. scoparia.

Worldwide Distribution: Atlantic-Mediterranean (SolerMembrives and Munilla, 2015).

GENUS: TRYGAEUS DOHRN, 1881

\section{T. communis Dohrn, 1881}

The species was reported by Kocak (2019) from Mordoğan.

Depth range: $0.5 \mathrm{~m}$.

Substrate: Cystoseira mediterranea Sauvageau, 1912. 
Worldwide Distribution: Mediterranean (SolerMembrives and Munilla, 2015).

FAMILY: ASCORHYNCHIDAE HOEK, 1881

GENUS: ASCORHYNCHUS G.O.SARS, 1877

\section{A. castelli (Dohrn, 1881)}

Synonyms: Barana castelli Dohrn, 1881

This species was reported by Kocak (2012) from Mordoğan.

Depth range: $10 \mathrm{~m}$.

Substrate: Posidonia oceanica (Linnaeus) Delile, 1813.

Worldwide Distribution: Atlantic-Mediterranean (SolerMembrives and Munilla, 2015).

SUPERFAMILY: PHOXICHILIDOIDEA G.O. SARS, 1891

FAMILY: PHOXICHILIDIIDAE G.O. SARS, 1891

GENUS: ANOPLODACTYLUS WILSON, 1878

\section{A. petiolatus (Kroyer, 1844)}

Synonyms: Phoxichilidium petiolatum Kroyer, 1844; Pallene attenuata Hodge, 1863; Phoxichilidium attennuata (Hodge, 1863); Phoxichilidium longicolle Dohrn, 1881; Phoxichilidium pygmaeum Hoek, 1881; Anoplodactylus longicollis (Dohrn, 1881); Anoplodactylus pygmaeus (Hoek, 1881); Anoplodactylus hedgpethi Bacescu, 1959; Anoplodactylus guyanensis Child, 1977.

This species was reported by Arnaud (1976) from Kalabak.

Depth range: $0.5 \mathrm{~m}$.

Substrate: E. elongata (as C. mediterranea).

Worldwide Distribution: Atlantic-Mediterranean (SolerMembrives and Munilla, 2015).

\section{A. pygmaeus (Hodge, 1864)}

Synonyms: Pallene pygmaea Hodge, 1864; Phoxichilidium pygmaeum (Hodge, 1864); Anoplodactylus exiguus (Dohrn, 1881); Phoxichilidium exiguum Dohrn, 1881; Halosoma derjugini Losina-Losinsky, 1929; Anoplodactylus derjugini (Losina-Losinsky, 1929)

This species was reported by Arnaud (1976) from Tuzla, Krapp et al. (2008) from Balıklıova, and Çinar et al. (2008) from Alsancak Harbour, Pasaport Harbour, and İnciraltı.

Depth range: $0.2-8 \mathrm{~m}$.

Substrate: M. galloprovincialis; P. oceanica.

Worldwide Distribution: Atlantic-Mediterranean (SolerMembrives and Munilla, 2015).

A. virescens (Hodge, 1864)
Synonyms: Orithyia globosa Goodsir, 1842; Phoxichilidium globosum Goodsir, 1842; Phoxichilidium virescens Hodge, 1864

This species was reported by Arnaud (1976) from Kalabak and Foça.

Depth range: $0.3-1 \mathrm{~m}$.

Substrate: C. crinata; Padina pavonia (Linnaeus) J.V. Lamouroux, 1816; E. elongata (as C. mediterranea).

Worldwide Distribution: Disrupted distribution (St. Paul I., Amsterdam, Mediterranean Sea including eastern and western basins) (Soler-Membrives and Munilla, 2015).

FAMILY: ENDEIDAE NORMAN, 1908

GENUS: ENDEIS PHILIPPI, 1843

\section{E. spinosa (Montagu, 1808)}

Synonyms: Chilophoxus spinosus Montagu, 1808; Endeis gracilis Philippi, 1843; Endeis laevis (Grube, 1871); Endeis vulgaris (Dohrn, 1881)

This species was reported by Krapp et al. (2008) from Foça (one juvenile specimen as E. cf. spinosa).

Depth range: $0.5 \mathrm{~m}$.

Substrate: C. crinita.

Worldwide Distribution: Atlantic-Mediterranean (SolerMembrives and Munilla, 2015).

SUPERFAMILY: NYMPHONOIDEA POCOCK, 1904

FAMILY: CALLIPALLENIDAE HILTON, 1942

GENUS: CALLIPALLENE FLYNN, 1929

C. emaciata (Dohrn, 1881)

Synonyms: Pallene emaciata Dohrn, 1881; Callipallene emaciata (Dohrn, 1881)

This species was reported by Arnaud (1976) and Krapp et al. (2008) from Foça.

Depth range: $0.5 \mathrm{~m}$.

Substrate: E. elongata (as C. mediterranea); C. crinita.

Worldwide Distribution: Atlantic-Mediterranean (SolerMembrives and Munilla, 2015).

\section{C. phantoma (Dohrn, 1881)}

Synonyms: Pallene phantoma Dohrn, 1881; Pallene phantopa Norman, 1908; Callipallene phantoma crinita Stock, 1952.

This species was reported by Arnaud (1976) from Urla.

Depth range: $0.7-1 \mathrm{~m}$.

Substrate: $P$. pavonia. 
Worldwide Distribution: Atlantic-Mediterranean (SolerMembrives and Munilla, 2015).

\section{C. spectrum (Dohrn, 1881)}

Synonyms: Pallene spectrum Dohrn, 1881 Urla.

The species was reported by Krapp et al. (2008) from

Depth Range: 0-1m.

Substrate: Stones; Cladocora cespitosa (Linnaeus, 1767).

Worldwide Distribution: Atlantic-Mediterranean (SolerMembrives and Munilla, 2015).

\section{C. tiberi (Dohrn, 1881)}

Synonyms: Pallene tiberii Dohrn, 1881; Callipallene emaciata tiberii (Dohrn, 1881)

This species was reported by Koçak (2016) from Urla.

Depth range: $0.5 \mathrm{~m}$

Substrate: C. mediterranea.

Worldwide Distribution: Atlantic-Mediterranean (SolerMembrives and Munilla, 2015).

FAMILY: NYMPHONIDAE WILSON, 1878

GENUS: NYMPHON FABRICIUS, 1794

\section{Nymphon gracile Leach, 1814}

Synonyms: Nymphon gallicum Hoek, 1881

This species was reported by Arnaud (1976) from Alsancak Harbour, and Çinar et al. (2008) from Pasaport Harbour and Alsancak Harbour.

Depth range: $0.2 \mathrm{~m}$.

Substrate: Boat hulls; M. galloprovincialis.

Worldwide Distribution: Atlantic-Mediterranean (SolerMembrives and Munilla, 2015).

\section{REFERENCES}

Arnaud, F. (1976). Sur quelques pycnogonides de Turquie et de la mer Egée (Méditerranée orientale). Acta Ecologica Iranica, 1(1), 68-71.

Arnaud, F. (1987). Les pycnogonides (Chelicerata) de Méditerranée: distribution écologique, bathymetrique et biogéographie. Mésogée, 47, 37-58.

Chimenz, C. (2000). Picnogonidi delle coste italiane: quadro delle conoscenze (Pycnogonida). Memorie della Società Entomologica Italiana, 78, 541-574.

Chimenz, C., Tosti, M. \& Cottarelli, V. (1993). Taxonomical and ecological observations on Pycnogonida from Apulian coasts (Southern Italy). Bollettino di Zoologia, 60, 339-347.

Çinar, M.E., Katağan, T., Koçak, F., Öztürk, B., Ergen, Z., Kocataş, A., Önen, M., Kirkim, F., Bakir, K., Kurt, G., Dağli, E., Açik, S., Doğan, A. \& Özcan, T. (2008). Faunal assemblages of the mussel Mytilus galloprovincialis in and around Alsancak Harbour (Izmir Bay, eastern Mediterranean) with

\section{DISCUSSION}

Ammothella longioculata is only known from the Mediterranean Sea. Spanish coast: Chafarinas Islands, Alboran Sea (Munilla \& Nieto, 1999). French coast: Nice (Arnaud, 1987). Tunisian coast: Tabarka (Arnaud, 1987). Italian coast: Levanto, Ligurian Sea (Faraggiana, 1940); Isola Lachea (Krapp, 1973); Apulian coast (Chimenz, et al., 1993); Costa d'Argento (Vignoli et al., 2006). Croatian coast: Rovinj, North Adriatic (Schüller, 1989). Israel coast: Tantura (Stock, 1958). Northern Cypriot coast: Famagusta Bay (Kocak and Katagan, 2007); Turkish coast: Gencelli cove (Krapp et al., 2008) (Figure 1).

A. longioculata is reported only one time from the Turkish waters up to date. The presence of this rare species is confirmed for the first time from the Izmir Bay, and the second time from Turkey in the present study.

With $A$. longioculata, the total number of species is raised to 19 in the İzmir Bay. These 19 different species of pycnogonids, out of a total number of 29 species in Turkey (Kocak, 2019), represent $65.5 \%$ of all known pycnogonid species. To date, a total number of 25 pycnogonid species are found in the Turkish Aegean Sea. Thus, the reported 19 species in the Izmir Bay represent $76.0 \%$ of the actual Turkish Aegean Sea species.

The family Ammotheidae is dominant with 9 species, followed by Callipallenidae (4 species), Phoxichilidiidae ( 3 species). Ammothella and Callipallene are the major genera (4 species), followed by Anoplodactylus (3 species).

All of the known pycnogonid species in İzmir Bay were reported in shallow water. Consequently, the studies conducted along the Izmir Bay coasts were based mainly on inshore samplings. Therefore, it is believed that deep-sea samplings in Izmir Bay and more detailed examinations along the Izmir Bay coasts will result in a more accurate count of pycnogonid species.

special emphasis on alien species. Journal of Marine Systems, 71(1-2), 1-17. DOI:10.1016/j.jmarsys.2007.05.004

Faraggiana, R. (1940). Pantopodi del Mare Ligure. Bollettino dei Musei di Zoologia e Anatomia Comparata di Torino, 48, 145-158.

Kocak, C. (2012). On the occurrence of Ascorhynchus castelli (Dohrn, 1881) (Arthropoda: Pycnogonida) In the Aegean Sea. Turkish Journal of Zoology, 36 (6), 831-834. DOI:10.3906/zoo-1202-6

Koçak, C. (2016). Callipallene tiberi (Dohrn, 1881) (Arthropoda, Pycnogonida): A Pycnogonid New for the Eastern Mediterranean. Turkish Journal of Fisheries and Aquatic Sciences, 16 (3),739-741. DOI:10.4194/1303-2712-v16_3_10

Kocak, C. (2019). A new record of the genus Trygaeus Dohrn, 1881 and species Trygaeus communis Dohrn, 1881 (Arthropoda, Pycnogonida) from Turkey (Eastern Mediteranean). Acta Adriatica, 60 (1):47-52. DOI:10.32582/aa.60.1.4 
Kocak, C. \& Katağan, T. (2007). First record of Ammothella longioculata (Faraggiana, 1940) (Pycnogonida, Ammotheidae) in the Cypriot coast (eastern Mediterranean). Marine Biodiversity Records, 1, 1-3. DOI:10.1017/S1755267207008755

Krapp, F. (1973). Pycnogonida from Pantelleria and Catania, Sicily. Beaufortia, 21, 55-74.

Krapp, F., Kocak, C. \& Katagan, T. (2008). Pycnogonida (Arthropoda) from the eastern Mediterranean Sea with description of a new species of Anoplodactylus. Zootaxa, 1686, 57-68. DOI:10.5281/zenodo.180521

Munilla, T. \& Nieto, D. (1999). Littoral pycnogonids from the Chafarinas Islands (Alboran Sea, western Mediterranean). Vie et Milieu, 49, 155161.
Schüller, S. (1989). Die pantopodenfauna von Rovinj (Nördliche Adria) und der Jahreszyklus einiger Arten. Bonner Zoologische Beiträge, 40, 285295.

Soler-Membrives, A. \& Munilla, T. (2015). PYCNOIB: Biodiversity and Biogeography of Iberian Pycnogonids. PLOS ONE, 10: 1-21. DOI:10.1371/journal.pone.0120818

Stock, J.H. (1958). Pycnogonida from the Mediterranean coast of Israel. Bulletin of the Research Council of Israel, 7B, 137-142.

Vignoli, V., Magari, V. \& Bernini, F. (2006). Preliminary study on the pycnogonids associated to photophilous algae from the 'Costa d'Argento' (Southern Tuscany) (Arthropoda Pycnogonida). Bollettino della Società Entomologica Italiana Genova, 138, 3-8. 